\title{
No relationship between the incidence of fentanyl-induced cough and smoking
}

\author{
Boris Pokhis, Hans-Bernd Hopf
}

Department of Anesthesia and Perioperative Medicine, Asklepios Klinik Langen, Academic teaching hospital of the Johann Wolfgang Goethe University Frankfurt am Main, Langen, Germany; corresponding author: h-b.hopf@asklepios.com

Received 17 September 2009; revised 22 September 2009; 23 September 2009.

\begin{abstract}
The purpose of the study was to investigate whether or not the incidence of cough after intravenous fentanyl depends on the patient's smoking state and the speed of injection. 530 ASA class I-III patients free of bronchial hyperreactivity and respiratory tract infection undergoing general anesthesia for elective surgery were randomized to 1.5 $\mathrm{g}^{\prime} \mathrm{kg}^{-1}$ fentanyl injected over 2,5 or $10 \mathrm{sec}$ or placebo via a peripheral intravenous cannula. The endpoint was cough within 5 min after completion of injection. Statistical evaluation was performed by factorial ANOVA and chi-square-test. Assuming around $25 \%$ smokers in our patient population calculated patient sample size was 340 per group. The study was terminated for futility after enrolment of $\mathbf{5 3 0}$ patients since an interims analysis yielded an incidence of cough of $2 \%$ both in smokers $(n=174)$ and nonsmokers $(n=356, p=$ 0,970 ), which was unrelated to the speed of injection and not different from placebo.
\end{abstract}

Keywords: Cough, fentanyl induced; smoking; fentanyl induced cough; cough; fentanyl; speed of injection

\section{INTRODUCTION}

Cough after intravenous fentanyl for induction of anesthesia has been overestimated for more than 15 years. The question of fentanyl-induced cough is clinically of considerable importance since several prophylactic measures haven been proposed to suppress cough after injection of fentanyl (2-7) all with specific possible side effects. We have shown in ASA I-III nonsmoking European patients that the incidence of cough after $1.5 \mathrm{gkg}^{-1}$ fentanyl is around $4 \%$ and not related to the speed of injection [1], much lower than previously assumed [2-9].

*Presented in part at the German anesthesia annual congress, Leipzig, May 9th, 2009.
Fentanyl induced cough is also believed to be much lower in smokers compared to nonsmokers ( $3 \%$ vs $13 \%$ ) with the incidence depending on the speed of injection [8]. We hypothesised, based on our own data in nonsmoking European patients that there would be no clinically relevant difference between smokers and nonsmokers in fentanyl-induced cough at least in a European population. Accordingly, to evaluate the influence of smoking on the incidence of fentanyl induced cough we performed a single blinded, randomized controlled study with peripheral intravenous injection of $1.5 \mathrm{~g} \mathrm{~kg}^{-1}$ bw fentanyl. In addition we hypothesizedd that in smokers the speed of injection is also not related to the incidence of cough.

\section{METHODS}

With institutional and ethical committee approval and after having obtained informed written consent 542 ASA class I-III patients without history of bronchial hyperreactivity and free of any upper respiratory tract infections scheduled for elective general, orthopedical, or gynecological surgery with general anesthesia were randomised (computer generated list, permuted blocks of 12). The study was performed between Oktober $1^{\text {th }} 2007$ and May $31^{\text {th }} 2008$. Patients received either $1.5 \mathrm{~g} \mathrm{~kg}^{-1}$ bw. fentanyl injected over 2,5 or 10 seconds or saline placebo $(\mathrm{NaCl} 0.9 \%)$ injected over $2 \mathrm{sec}$. The study was carried out in a single blinded design i.e. patients were blinded concerning drug and speed of injection. Patient allocation was performed consecutively in the anesthesia pre-evaluation ambulance by the attending anaesthesiologist according to numbers generated by the computer generated list.

During the preparation of our previous study all anesthesia nurses $(\mathrm{n}=7)$ had been trained by means of a stop watch in delivering the three predefined injection velocities. After placement of the routine monitors (noninvasive blood pressure, ecg, pulse oximetry) the anesthesia nurse in charge injected the assigned drug within the assigned time controlled by a stop watch. After completion of injection patients were observed for 5 minutes. Througout the $5 \mathrm{~min}$ study period all patients were preoxygenated $\left(6 \mathrm{lmin}^{-1}\right.$ flow 
$\mathrm{O}_{2}$, semi-closed system with the expiration valve of the anesthesia circuit being set to $5 \mathrm{~cm}$ of water). Drugs were always injected at room temperature via a periphereal intravenous cannula at the back of the right or left hand in a running infusion of Ringer's lactate.

Primary endpoint was cough within 5 min after completion of injection. Cough was defined as any tussive reaction not consistent with normal quite breathing. Primary outcome was assessed by the attending anaesthesiologist. Secondary endpoints were nausea and vomiting or any other complaints of the patients within $5 \mathrm{~min}$ after completion of injection.

Basic assumptions were set as in our previous study: a $5 \%$ incidence of fentanyl-induced cough in the fentanyl and of $0 \%$ in the placebo group, an alpha error of 0.01 and a ßeta error of 0,05 resulting in 340 patients per group [10]. Assuming a portion of $25 \%$ smokers in our local population an interim analysis was planned after enrolment of 500 patients, when in all likelihood enough (more than 100) smokers were included into the study protocol to allow statistically meaningful results.

Statistical evaluation was performed with an analysis of variance for continuous and the chi-square-test for categorical variables. We compared the incidence of cough between smokers and nonsmokers in the whole group and the incidence of cough between fentanyl and placebo within the smoking and the non-smoking groups, respectively.

Our null hypothesis was that there were no significant differences in the incidence of cough between smokers and nonsmokers and between the fentanyl groups and saline placebo in the smoking and non-smoking group, respectively. After adjusting for multiple testing (bonferronis method) the null hypothesis was rejected and statistical significance assumed with a p value less than 0.001 .

\section{RESULTS}

The flow chart of the patients of our study is shown in Figure 1. Overall, from 542 patients enrolled, 530 received the allocated medication. Eleven patients did not present

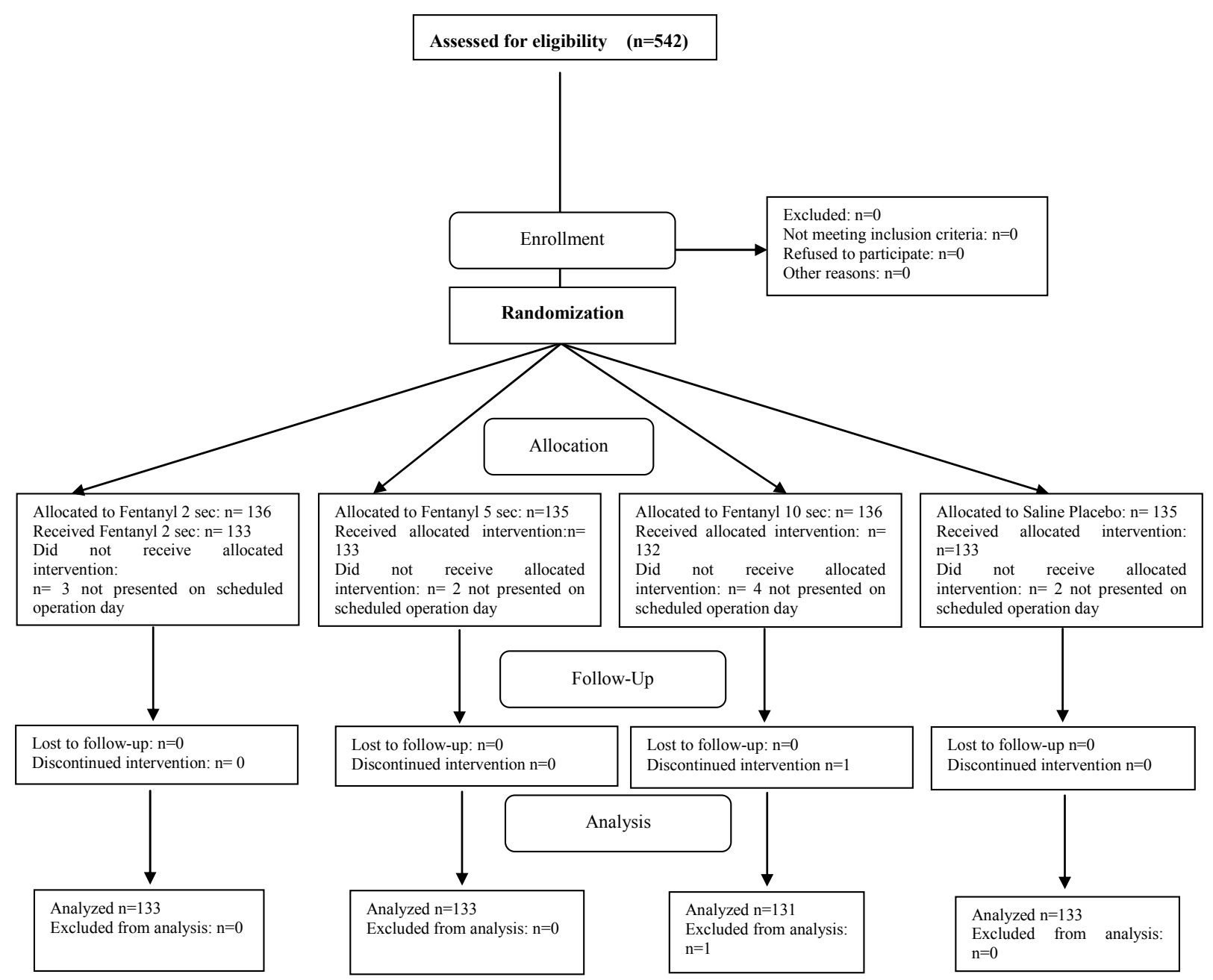

Figure 1. The consort E-flowchart. 
at the operation day scheduled, equally distributed in the four groups. In one patient in the fentanyl $10 \mathrm{sec}$ group the syringe with the study medication was dislocated during the injection. Therefore the patient was excluded from evaluation. Thus, enrolment, allocation and follow up of the patients were highly conformable to the study protocol.

The study was terminated for futility after enrolment of 530 patients since the planned interims analysis yielded an incidence of cough in $4 / 174$ patients $(=2 \%)$ in the smoking and of $8 / 356$ patients $(=2 \%)$ in the non-smoking group $(p=0.970)$, Table 1 shows the results of the three injection velocities in the smoking, non-smoking and overall patient groups compared to placebo respectively. There were no differences in fentanyl induced cough within the three fentanyl groups of different injection velocities neither when comparing between the fentanyl groups nor when comparing the fentanyl groups to placebo.

Only one patient in the fentanyl $2 \mathrm{sec}$ and one patient in the $5 \mathrm{sec}$ group (in the smoker group) reported nausea during the study period. No patient suffered from vomiting during the study period.

Demographic data (age, height, weight, distribution of sex) and ASA class did not differ between groups.

\section{DISCUSSION}

The main finding of our study is that the incidence of cough after $1.5 \mathrm{~g} \mathrm{~kg}^{-1}$ fentanyl is $2 \%$ both in smokers and nonsmokers. Within the smoking and non-smoking group it was also not related to the speed of injection and not different from saline placebo. Thus, this study confirmed our previous findings of a clinically irrelevant incidence of fentanyl induced cough in European people also in smokers.

Our results emerged when confounding variables such as patient population, different doses of fentanyl or speed or site of injection either were strictly controlled or eliminated. In addition, our study is the first, which compared in smokers different injection velocities not only between the fentanyl groups but also to saline placebo.

As in our previous study in non-smoking patients [1] we have choosen the $1.5 \mathrm{~g} \mathrm{~kg}^{-1}$ dose of fentanyl, because it is the routine used induction dose in $>90 \%$ of our patients. While even $7 \mathrm{~g} \mathrm{~kg}^{-1}$ fentanyl injected in a periphereal vein over 1 second evoked cough in only 1/37 patients in an European population [8], two studies in an asiatic/chinese population testing an identical dose of 1.5 $\mathrm{g} \mathrm{kg}^{-1}$ fentanyl found much higher incidences of up to 28 $\%[6,9]$, suggesting a race dependence.

Regardless of the smoking status, injection velocity did not influence the incidence of cough and was considerably lower than previously reported [6]. Since, compared to our study, the dose of fentanyl as well as the site of injection were identical, other reasons must be responsible. First, in the study of Lin et al. [6] the speed of injection (2, 15 and 30 seconds) was considerably slower than in our study (2, 5 and 10 seconds). Assuming any relation between the incidence of cough and the speed of injection a slower injection velocity should result in a lower incidence of cough. However, compared to our results the incidence of cough in the study by Lin was greater with slower injection velocities, excluding any possible relationship. Second, the study of Lin et al [6] and ours differed in the substantially longer postinjection observation period in our study (30 seconds in the study by Lin et al. vs. 5 minutes in our study protocol). Since a longer observation period in all likelihood should result in a higher rather than a lower incidence of cough by chance alone this also can be excluded as an explanation for the higher incidence of cough in the study by Lin et al. Thus racial differences of the subjects studied (Chinese vs. European people) seemed to be the most likely explanation for the different incidences of

Table 1. Incidence of cough after intravenous injection of fentanyl or placebo( $n=530)$.

\begin{tabular}{|c|c|c|c|c|}
\hline & $\begin{array}{c}\text { Fentanyl } 2 \sec (n=133) \\
\text { Cough yes/no } \\
(\%)\end{array}$ & $\begin{array}{c}\text { Fentanyl } 5 \sec (n=133) \\
\text { Cough yes/no } \\
(\%)\end{array}$ & $\begin{array}{c}\text { Fentanyl 10 sec } \\
(\mathrm{n}=131) \\
\text { Cough yes/no } \\
(\%)\end{array}$ & $\begin{array}{c}\text { Placebo }(n=133) \\
\text { Cough yes/no } \\
(\%)\end{array}$ \\
\hline \multirow[t]{3}{*}{ Smoker $(n=174)$} & $1 / 37$ & $1 / 44$ & $1 / 46$ & $1 / 43$ \\
\hline & $(2,6 \%)$ & $(2,2 \%)$ & $(2,1)$ & $(2,2 \%)$ \\
\hline & \multicolumn{4}{|c|}{$p=0,999$} \\
\hline \multirow[t]{3}{*}{ Nonsmoker $(\mathrm{n}=356)$} & $3 / 92$ & $2 / 86$ & $1 / 84$ & $2 / 86$ \\
\hline & $(3,1 \%)$ & $(2,2 \%)$ & $(1,0 \%)$ & $(2,2 \%)$ \\
\hline & \multicolumn{4}{|c|}{$\mathrm{p}=\mathbf{0 , 8 4 8}$} \\
\hline \multirow[t]{3}{*}{ Total $(n=530)$} & $4 / 129$ & $3 / 130$ & $2 / 129$ & $3 / 130$ \\
\hline & $(3,0 \%)$ & $(2,2 \%)$ & $(1,5 \%)$ & $(2,2 \%)$ \\
\hline & \multicolumn{4}{|c|}{$\mathrm{p}=\mathbf{0 , 8 8 4}$} \\
\hline
\end{tabular}

Values shown are numbers. Values in parenthesis represent percentage of patients with cough. P-values refer to the chi-square-test. 
cough between the study by Lin et al. compared to our study. In fact, in an asiatic or chinese population $[2,3,6,7,9]$ intravenous injection of fentanyl in doses ranging from 2-5 $\mathrm{g} \mathrm{kg}^{-1}$ given within 1-5 seconds evoked cough in $28-65 \%$ in contrast to $3 \%$ incidence after 75 $\mathrm{g} \mathrm{kg}^{-1}$ given within 1 second in an European population [8].

That in our study cough was also evoked after saline placebo injection in all likelihood is the result of our considerably longer post-injection observation period ( 5 minutes) in conjunction with preoxygenation by face mask, both increasing the chance for cough either by time alone or by inconvenience of the mask regardless of the drug injected. Consistent with this assumption, in the placebo groups cough occurred in 2 patients after 1,5 and 3 minutes in the nonsmokers and after 4 minutes in one smoker, respectively, in contrast to the fast ( $<1$ minute) response after intravenous fentanyl.

In summary, in this study in an European population the incidence of cough after intravenous injection of fentanyl was 1) not different between smokers and nonsmokers, 2) unrelated to the speed of injection within the smoking and non-smoking group, and 3) not different from saline placebo. Therefore in a routine clinical setting fentanyl-indued cough is a rare event without any need for prophylaxis.

\section{REFERENCES}

[1] U. Schäpermeier, H. B. Hopf, (2008) Fentanyl-induced cough does not depend on injection speed: A randomised study. Acta Anaesthesiol Scand, 52, 1071-5.

[2] P. W. Lui, C. H. Hsing, Y. C. Chu, (1996) Terbutaline inhalation suppresses fentanyl-induced coughing. Can J Anaesth, 43, 1216-9.

[3] A. Agarwal, A. Azim, S. Ambesh, et al., (2003) Salbutamol, beclomethasone or sodium chromoglycate suppress coughing induced by iv fentanyl. Can J Anaesth, 50, 297-0.

[4] C. S. Lin, W. Z. Sun, W. H. Chan, C. J. Lin, H. M. Yeh, M. S. Mok, (2004) Intravenous lidocaine and ephedrine, but not propofol, suppress fentanyl induced cough. Can J Anaesth, 51, 654-9.

[5] C. K. Pandey, M. Raza, R. Ranjan, et al., (2004) Intravenous lidocaine suppresses fentanyl-induced coughing: a double-blind, prospective, randomized placebo-controlled study. Anesth Analg, 99, 1696-8.

[6] J. A. Lin, C. C. Yeh, M. S. Lee, C. T. Wu, S. L. Lin, C. S. Wong, (2005) Prolonged injection time and light smoking decrease the incidence of fentanyl-induced cough. Anesth Analg, 101, 670-4.

[7] H. C. Horng, C. S. Wong, K. N. Hsiao, B. K. Huh, C. P. Kuo, C. H. Cherng, C. T. Wu, (2007) Pre-medication with intravenous clonidine suppresses fentanyl-induced cough. Acta Anaesthesiol Scand, 51, 862-865.

[8] H, Böhrer, F. Fleischer, P. Werning, (1990) Tussive effect of a fentanyl bolus administered through a central venous catheter. Anaesthesia, 45, 18-21.

[9] W. T. Phua, B. T. Teh, W. Jong, T. L. Lee, W. A. Tweed, (1991) Tussive effect of a fentanyl bolus. Can J Anaesth, 38, 330-4.

[10] S. J. Pocock, (1998) Clinical trials: A practical aproach. Chichester New York Brisbanae Toronto Singapore: John Wiley \& Sons, Chapter 9, 123-141. 